Check for updates

Cite this: RSC Adv., 2019, 9, 30033

Received 18th July 2019

Accepted 4th September 2019

DOI: $10.1039 / c 9 r a 05526 f$

rsc.li/rsc-advances

\section{Metabolic profiling reveals the heterogeneity of vascular endothelial function phenotypes in individuals at extreme cardiovascular risk $\dagger$}

\author{
Baoyu Mao, † $^{\mathrm{a}}$ Yanshan Yi, + $^{\mathrm{a}}$ Qiuyan Mo, ${ }^{\mathrm{a}}$ Chunxiu Yang ${ }^{\mathrm{a}}$ and Qiuan Zhong (DD *ab
}

Maladapted vascular endothelial metabolism in the context of endothelial function differing in phenotype remains unknown, which limits our understanding of the heterogeneous pathogenesis of atherosclerotic cardiovascular disease (CVD). This study aimed to profile serum metabolic alterations of different vascular endothelial function phenotypes in asymptomatic adults at extreme cardiovascular risk. In addition to 12 CVD patients, 103 individuals free of CVD were categorized as having normal endothelial function (NEF) ( $n=30)$, cardiovascular risk-promoting endothelial function (PEF) $(n=18)$, cardiovascular risk-resistant endothelial function (REF) ( $n=25)$, and vulnerable endothelial function (VEF) $(n=30)$. Serum metabolic profiles were detected using gas chromatography time-of-flight/mass spectrometry and multivariate statistics. Compared to the NEF group, a total of 17, 17, 22, and 13 differential metabolites were identified in the PEF, REF, VEF, and CVD groups, respectively. Of the altered metabolic pathways, multiple pathways were consistent between the PEF and CVD groups, including pyrimidine metabolism, starch and sucrose metabolism, aminoacyl-tRNA biosynthesis, arginine and proline metabolism, and D-glutamine and D-glutamate metabolism. Notably, a relative increase in low-calorie sugar in galactose metabolism was exclusively found in the REF group, and a relative increase in the ratio of acetyl-COA to COA was suggested in the VEF group based on elevated butanoate metabolism and reduced pantothenate and COA biosynthesis. Our findings clearly indicate distinct metabolic patterns across groups with heterogeneous vascular endothelial function in the context of extreme cardiovascular risk, and improve our understanding of the pathogenic heterogeneity of early CVD in asymptomatic populations.

\section{Introduction}

More attention is paid to populations at extreme cardiovascular risk. In fact, approximately $35 \%$ of individuals at high cardiovascular risk are less likely to develop atherosclerotic cardiovascular disease (CVD) events, while the risk of CVD events is elevated from $12 \%$ to $21 \%$ in individuals at low cardiovascular risk, ${ }^{\mathbf{1}, 2}$ suggesting a heterogeneous pathogenesis in atherosclerotic processes. Previous studies have also reported outlier individuals who did not have the expected number of CVD events according to their specific cardiovascular risk level; $;^{3-6}$ however, the underlying mechanisms are largely unclear. Indeed, as an outcome of a multistage, complex and chronic

\footnotetext{
${ }^{a}$ Guangxi Colleges and Universities Key Laboratory of Prevention and Control of Highly Prevalent Diseases, Guangxi Medical University School of Public Health, 22 Shuangyong Road, Nanning 530021, China. E-mail: qazhong@gxmu.edu.cn

${ }^{b}$ Department of Epidemiology, Guangxi Medical University School of Public Health, 22 Shuangyong Road, Nanning 530021, China

$\dagger$ Electronic supplementary information (ESI) available. See DOI: 10.1039/c9ra05526f

$\$$ The first two authors contributed equally to this study.
}

pathological process, different CVD types with notable pathophysiological differences exist. ${ }^{7}$ Thus far, studies have remained focused on the clinical stage of CVD, which may lead to an incomplete understanding of the heterogeneity in atherogenesis.

Vascular endothelial dysfunction, characterized mainly by the loss of nitric oxide (NO) bioavailability, is an initial manifestation in the pathological development of atherosclerotic CVD. ${ }^{8-10}$ More importantly, dysfunctional endothelium serves as a pathological indicator in diseased vasculature through metabolic signals. ${ }^{11}$ Therefore, knowledge of the endogenous metabolic profiles related to endothelial function under different risk profiles may be crucial to understanding the heterogeneity of atherogenesis. Increasing cardiovascular metabolomics evidence has recently emerged from patients with carotid atherosclerotic plaques and coronary artery diseases. ${ }^{\mathbf{1 2 - 1 5}}$ However, metabolic profiling of early cardiovascular pathology, i.e., vascular endothelial dysfunction, is still poorly understood in the asymptomatic population.

In this study, we aim to profile metabolic changes relevant to the heterogeneous vascular endothelial functions in the serum of asymptomatic individuals at extreme cardiovascular risk 
using an untargeted metabolomics approach based on gas chromatography time-of-flight/mass spectrometry (GC-TOF/MS) with high resolution and detection sensitivity. ${ }^{\mathbf{1 6 , 1 7}}$ Additionally, the present analyses seek to explore the potential mechanism pathways involved in heterogeneous atherogenesis from metabolic clues.

\section{Methods}

\section{Study population}

A total of 899 subjects aged 20 to 82 years including 865 asymptomatic individuals and 34 CVD patients identified by written medical records were recruited from Rongan and Rongshui of Guangxi, China in 2015. Data were generated by a cardiovascular health survey including an interview questionnaire (age, sex, ethnicity, cigarette smoking, history of CVD and medication use), physical examination (brachial artery flow-mediated dilation (FMD), body mass index (BMI), heart rate and blood pressure) and fasting serum collection (metabolites, total cholesterol, triglycerides, low-density lipoprotein (LDL) cholesterol, high-density lipoprotein (HDL) cholesterol, glucose and C-reactive protein (CRP)). The health survey and all experiments with blood samples in this study were performed in strict accordance with the Declaration of Helsinki Principles. All participants provided informed consent, and the study protocols were approved by the Medical Ethics Committee of Guangxi Medical University.

\section{Cardiovascular risk estimation}

The cardiovascular risk for asymptomatic participants aged 3074 years was estimated using the general formula of the sexspecific 10 year Framingham risk score (FRS), which includes age, total cholesterol, HDL cholesterol, treated or untreated systolic blood pressure, current smoking and diabetes. ${ }^{18}$ The estimated cardiovascular risk was categorized as low (FRS $\leq$ $6 \%$ ), moderate (FRS $>6 \%$ and $\leq 20 \%$ ), or high (FRS $>20 \%$ ).

\section{Vascular endothelial function assessment}

Vascular endothelial function was assessed by brachial artery FMD. All participants abstained from using vasoactive medications, smoking, alcohol use, caffeine consumption, or eating a high-fat diet the day before the measurement. After resting for at least $15 \mathrm{~min}$, measurements were performed using a UNEX EF38G high-resolution ultrasound system (UNEX Corporation, Nagoya, Japan) in a quiet room at a comfortable temperature. Details of the FMD measurement have been described elsewhere. ${ }^{19}$ The brachial FMD is expressed as the percent increase in maximum diameter after reactive hyperemia relative to the baseline brachial artery diameter. Endothelial function was categorized as good (FMD $\geq 10 \%$ ), moderate (FMD $\geq 6 \%$ and $<10 \%$ ), or dysfunctional (FMD $<6 \%$ ).

\section{Definition of vascular endothelial phenotypes}

Four endothelial phenotypes were set up in asymptomatic participants. Normal endothelial function (NEF) was defined as having low cardiovascular risk and good endothelial function, cardiovascular risk-promoting endothelial function (PEF) was defined as having high cardiovascular risk and dysfunctional endothelial function, cardiovascular risk-resistant endothelial function (REF) was defined as having high cardiovascular risk but good endothelial function, and vulnerable endothelial function (VEF) was defined as having low cardiovascular risk but dysfunctional endothelial function.

For the metabolomics analysis, 30 individuals with NEF, 18 individuals with PEF, 25 individuals with REF, and 30 individuals with VEF were sampled with overall matching for age and sex from 680 candidates. Additionally, 12 patients with coronary artery disease were included for comparison of metabolites, in order to comprehensively assess the distinct metabolic patterns of endothelial function (ESI Fig. $1 \dagger$ ). All subjects enrolled in the metabolomics analysis were free of any agent use for at least 3 days prior to the survey.

\section{Sample preparation for GC-TOF/MS}

A $100 \mu \mathrm{L}$ aliquot of serum was transferred into a $1.5 \mathrm{~mL}$ centrifuge tube and vortexed for $10 \mathrm{~s}$ after adding $0.35 \mathrm{~mL}$ of methanol and $20 \mu \mathrm{L}$ of $\mathrm{L}$-2-chlorophenylalanine as an internal standard. The mixture was centrifuged at $13000 \mathrm{rpm}$ for $15 \mathrm{~min}$ at $4{ }^{\circ} \mathrm{C}$. Then, $0.4 \mathrm{~mL}$ of supernatant was transferred into a $2 \mathrm{~mL}$ silylated glass vial and dried in a vacuum concentrator without heating. A $50 \mu \mathrm{L}$ aliquot of methoxy amination hydrochloride (20 $\mathrm{mg} \mathrm{mL}^{-1}$ in pyridine) was subsequently added to the dried extracts, followed by incubation in an oven for $30 \mathrm{~min}$ at $80{ }^{\circ} \mathrm{C}$ after mixing gently. Then, $70 \mu \mathrm{L}$ of bistrifluoroacetamide (containing $1 \%$ TCMS, v/v) was added to each mixture and incubated at $70{ }^{\circ} \mathrm{C}$ for $2 \mathrm{~h}$. Finally, the mixture was cooled to room temperature, followed by the addition of $10 \mu \mathrm{L}$ of FAMEs (standard mixture of fatty acid methyl esters, C8-C16: $1 \mathrm{mg}$ $\mathrm{mL}^{-1}$; C18-C24: $0.5 \mathrm{mg} \mathrm{mL}^{-1}$ in chloroform) for GC-TOF/MS analysis.

\section{GC-TOF/MS analysis and data processing}

GC-TOF/MS was performed using an Agilent 7890 gas chromatograph system (Agilent, USA) coupled with a Pegasus HT time-of-flight mass spectrometer (LECO, Saint Joseph, MI, USA). The system utilized a DB-5MS capillary column coated with 5\% diphenyl cross-linked with 95\% dimethylpolysiloxane $(30 \mathrm{~m} \times 250 \mu \mathrm{m}$ inner diameter, $0.25 \mu \mathrm{m}$ film thickness; J\&W Scientific, Folsom, CA, USA). A $1 \mu \mathrm{L}$ aliquot was injected in splitless mode, using helium as the carrier gas, with a $3 \mathrm{~mL} \mathrm{~min}{ }^{-1}$ front inlet purge flow and a $1 \mathrm{~mL} \mathrm{~min}{ }^{-1}$ gas flow rate through the column. The initial temperature of the column was kept at $50{ }^{\circ} \mathrm{C}$ for $1 \mathrm{~min}$; it was then raised to $310{ }^{\circ} \mathrm{C}$ at a rate of $20{ }^{\circ} \mathrm{C} \min ^{-1}$ and maintained for $6 \mathrm{~min}$. The temperatures of injection, the transfer line and the ion source were 280,270 and $220^{\circ} \mathrm{C}$, respectively. The energy was set at $70 \mathrm{eV}$ in electron impact mode. The mass spectrometry data were acquired at a rate of 20 spectra per second after a solvent delay of $366 \mathrm{~s}$ with a mass-to-charge $(\mathrm{m} / \mathrm{z})$ range of 50-500 in full-scan mode.

Raw data were obtained using Chroma TOF 4.3X software (LECO, Saint Joseph, MI, USA) and the LECO-Fiehn Rtx5 
database after raw peak extraction, baseline data filtering and calibration, peak alignment, deconvolution analysis, peak identification, and peak area integration. The raw peaks were retained after further filtering by the interquartile range denoising method. Then, for missing values in the raw data, a simulation method with half of the minimum value was used to fill up the dataset. Finally, the remaining data were processed using an internal standard normalization method. The LECO/ Fiehn Metabolomics Library was used to evaluate the accuracy of compound identification via a similarity value. The identified metabolites were further verified by searching the PubChem Compound database (http://pubchem.ncbi.nlm.nih.gov), the Kyoto Encyclopedia of Genes and Genomes (KEGG) database (http://www.genome.jp/kegg), and the Human Metabolome database (http://www.hmdb.ca).

\section{Statistical analysis}

In total, 4 comparisons, including PEF versus NEF, REF versus NEF, VEF versus NEF and CVD versus NEF, were performed in this study. Briefly, after importing the metabolic data, including peak number (RT and $\mathrm{m} / \mathrm{z}$ pairs), sample name (observation) and normalized peak area (variable), the SIMCA 14.1 software package (Umetrics, Umea, Sweden) was used for principal component analysis (PCA) and orthogonal projections to latent structures discriminant analysis (OPLS-DA). The PCA was used to characterize the overall distribution of the data matrix for all groups. A supervised OPLS-DA was used to discriminate the different metabolites between the comparison groups. The models were evaluated using 7 -fold cross validation and permutation tests.

The metabolites that differed between comparison groups were selected according to the variable importance in the projection (VIP) value (VIP value $>1.0$ ) and Student's $t$ test $(P$ value $<0.05)$. The relevant metabolic pathways of the differential metabolites were searched via databases including the KEGG and the NIST (http://www.nist.gov/ index.html). The differential metabolites were further exported to the online MetaboAnalyst (http:// www.metaboanalyst.ca) for pathway analysis that integrated enrichment analysis and pathway topology analysis. Moreover, receiver operating characteristic (ROC) analysis was performed using STATA version 13.1 (StataCorp LP, College Station, TX, USA) to evaluate the accuracy of the discriminatory ability of differential metabolites.

\section{Results}

\section{Characteristics of the study participants}

The average FMD of the participants was $12.8 \%$ for NEF, $4.4 \%$ for PEF, $11.8 \%$ for REF, $4.0 \%$ for VEF, and $7.8 \%$ for CVD. Generally, individuals with PEF were more likely to have higher percentages or levels of current smoking, BMI, systolic blood pressure, and serum triglyceride, but lower levels of serum HDL cholesterol. Individuals with REF were more likely to have higher heart rates, serum total cholesterol, and serum LDL cholesterol. Individuals with VEF were more likely to have lower percentages or levels of current smoking, BMI, systolic blood pressure, heart rates, serum CRP, serum total cholesterol, serum triglyceride, serum LDL cholesterol, and diabetes but a higher level of serum HDL cholesterol. CVD patients were more likely to be older, have higher serum CRP, and have diabetes (ESI Table 1†).

\section{Compound profiles by GC-TOF/MS}

The typical total ion chromatograms (TICs) of the serum samples from the five groups are shown in Fig. 1. A total of 126 compounds were ultimately identified according to the LECO/ Fiehn Metabolomics Library, the majority of which were endogenous metabolites including amino acids, organic acids, fatty acids, carbohydrates and nucleosides. Based on the PCA score plot, all samples were within the 95\% Hotelling's $T$ squared ellipse, generally indicating separable clusters in the metabolic profiles of the five groups, except for between PEF and CVD (Fig. 2).

\section{Differential metabolites for different endothelial phenotypes}

In this study, clear separations are shown in all comparisons by the OPLS-DA models (Fig. 3A-D), with good fitness and prediction indicated by $R^{2} Y$ values ranging from 0.925 to 0.983 and $Q^{2}$ values ranging from 0.697 to 0.892 (ESI Table $2 \dagger)$. For all comparisons, the permutation tests with low values of $R^{2}$ and $Q^{2}$ indicated robustness and a low risk of overfitting for the OPLS-DA models (Fig. 3E-H).

Altogether, 42 unique differential metabolites with VIP values $>1.0$ and $P$ values $<0.05$ are shown in Table 1 . Compared to NEF, a total of $17,17,22$, and 13 metabolites were significantly altered in PEF, REF, VEF, and CVD, respectively. Among the differential metabolites, there were 6 and 11 metabolites for PEF, 7 and 10 metabolites for REF, 9 and 13 metabolites for VEF, and 1 and 12 metabolites for CVD that increased (fold change value $>1$ ) and decreased (fold change value $<1$ ), respectively. Moreover, 7, 6, 8, and 3 exclusive metabolites were found for PEF, REF, VEF, and CVD, respectively.

\section{Altered metabolic pathways related to different endothelial phenotypes}

The significantly altered metabolic pathways were revealed for the different endothelial phenotypes (Fig. 4). The same metabolic pathways were altered in the PEF and CVD groups, including pyrimidine metabolism, starch and sucrose metabolism, aminoacyl-tRNA biosynthesis, arginine and proline metabolism, and D-glutamine and D-glutamate metabolism. Additionally, alanine, aspartate and glutamate metabolism, and pyruvate metabolism were also perturbed in the patients with CVD. Meanwhile, galactose metabolism was exclusively disturbed in the REF group, while 4 metabolic pathways were clearly altered in the VEF group, including 2 unique pathways, namely, pantothenate and CoA biosynthesis, and butanoate metabolism.

The differential metabolites involved in the significantly altered metabolic pathways are shown in ESI Table 3.† For 

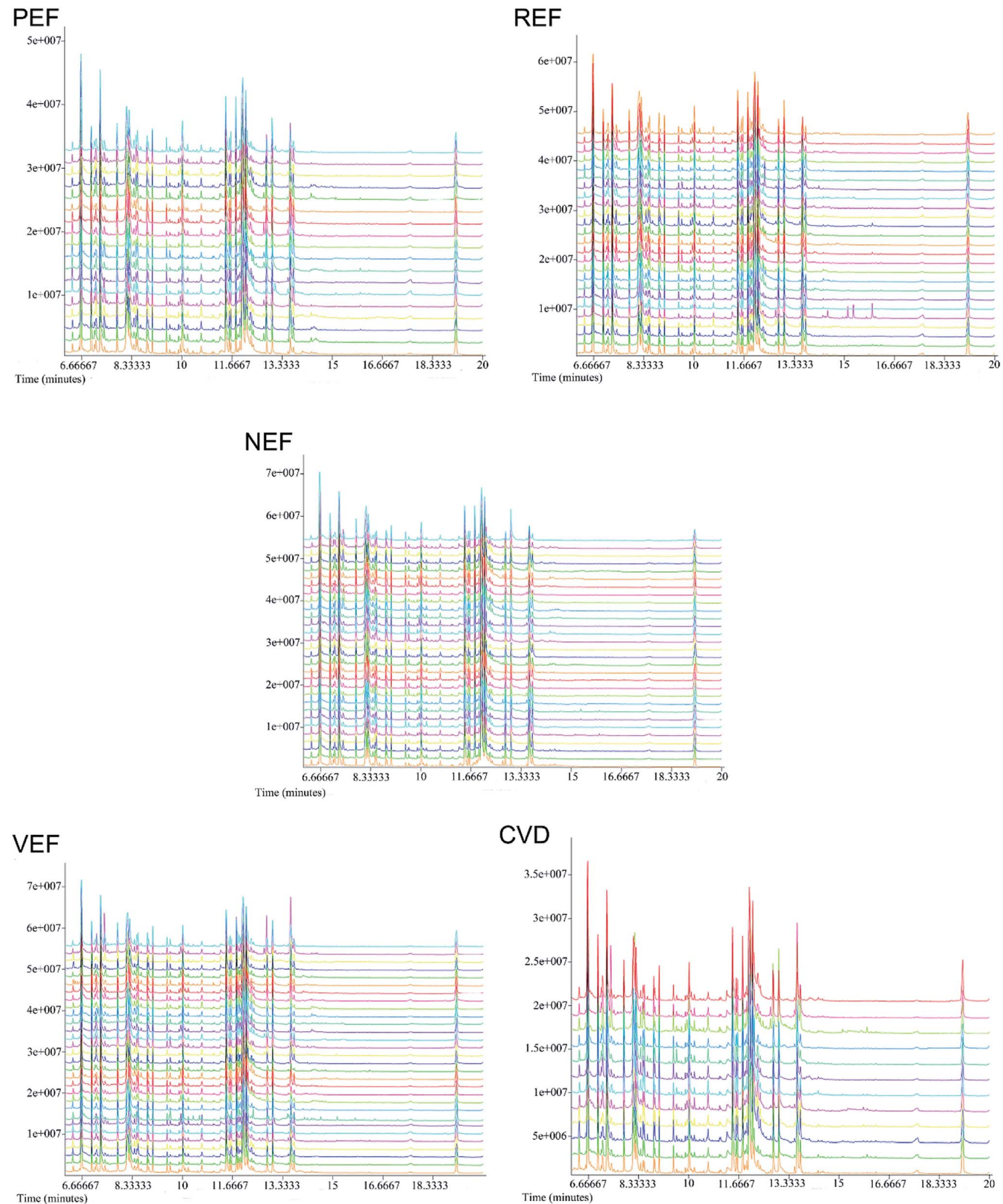

Fig. 1 Representative GC-TOF/MS total ion chromatograms of the samples from the NEF, PEF, REF, VEF and CVD groups. GC-TOF/MS, gas chromatography time-of-flight/mass spectrometry; NEF, normal endothelial function; PEF, cardiovascular risk-promoting endothelial function; $\mathrm{REF}$, cardiovascular risk-resistant endothelial function; VEF, vulnerable endothelial function; CVD, cardiovascular disease.

different comparisons, the ROC analyses showed that the area under the curve (AUC) values of the differential metabolites ranged from 0.734 to 1.000 in various metabolic pathways.
Moreover, the AUC values were not significantly different after further adjustment for age and sex in the models $(P$ values $>$ $0.05)$. 


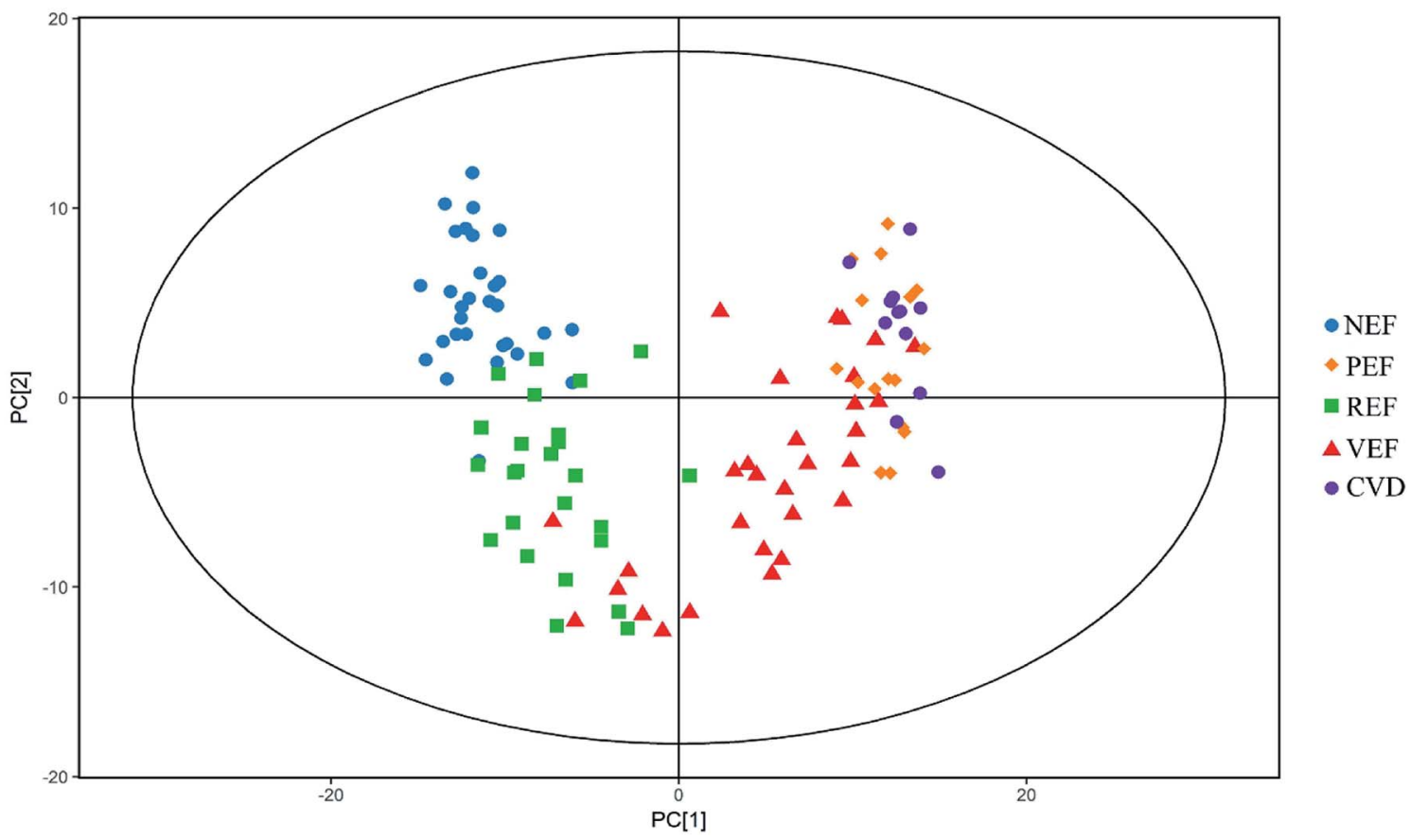

Fig. 2 The PCA score plots of different endothelial function groups and the CVD group. PCA, principal components analysis; NEF, normal endothelial function; PEF, cardiovascular risk-promoting endothelial function; REF, cardiovascular risk-resistant endothelial function; VEF, vulnerable endothelial function; CVD, cardiovascular disease.

\section{Discussion}

In this metabolomics analysis of serum samples of 103 asymptomatic subjects and $12 \mathrm{CVD}$ patients, some metabolites were altered across all of the different endothelial phenotypes, while other metabolites were exclusive to specific endothelial phenotypes, such as monoolein and 1-monopalmitin for the PEF phenotype, tagatose and lyxose for the REF phenotype, and valine and 4hydroxybutyrate for the VEF phenotype. Furthermore, the altered metabolites were enriched in differential metabolic pathways, suggesting distinct metabolic patterns in response to a specific endothelial function status.

Impaired endothelial function was associated with higher cardiovascular risk in asymptomatic adults, ${ }^{20,21}$ which was mechanistically involved via pathological changes such as decreased NO synthesis, increased oxidative stress, and increased inflammation. ${ }^{22}$ Urea and glutamic acid, in arginine and proline metabolism, are connected to the ornithine cycle and the deamination of glutamic acid. Glutamic acid fluxes into the ornithine cycle as citrulline via deamination and subsequently synthesizes arginine in the cytoplasm; ${ }^{23,24}$ arginine is then converted to urea, which contributes to the excretion of excess ammonia. Not just an intermediate for ammonia excretion, arginine can also function as a precursor and immediately produce NO under the catalysis of endothelial NO synthase (eNOS). In this study, elevated urea and deceased glutamic acid may indicate active deamination in the form of urea and a relative reduction of NO synthesis in the subjects with PEF. Interestingly, elevation of urea has been proven to cause endothelial dysfunction via inducing reactive oxygen species (ROS) production in uremic mice. ${ }^{25}$ Uric acid and 3-aminoisobutyric acid were downregulated in the PEF group compared with the NEF group, suggesting increased oxidative stress according to in vitro and in vivo evidence. ${ }^{26,27}$ Additionally, tryptophan, an indicative essential amino acid for protein synthesis, was remarkably deficient in the PEF group. An inverse association between circulating tryptophan and inflammation has been confirmed in CVD patients by epidemiological studies. ${ }^{28,29}$

Active catabolism of pyrimidines was suggested in the PEF group, as shown by upregulated urea and downregulated 3aminoisobutyric acid and thymidine, since urea is one of the main catabolic products of pyrimidine metabolism. ${ }^{30}$ As a terminal oxidation product of purines, a remarkable decrease in uric acid suggested a reduction of purine synthesis in the PEF groups. The noteworthy metabolite reductions in the purine and pyrimidine pathways have been confirmed in atherosclerotic plaques. ${ }^{31}$ Compared with the NEF group, the PEF group had invalid biosynthesis of aminoacyl-tRNA, which is crucial for growing polypeptide chains, ${ }^{32}$ suggesting that protein synthesis may be somewhat impaired in the PEF group. Additionally, as precursors of glucose, both sucrose and fructose were at low levels in the PEF, indicating an insufficient energy state. Notably, monoolein and 1-monopalmitin, both monoacylglycerol species of triglycerides, were somewhat elevated in the PEF group; similar circulating triglyceride species, instead of total triglycerides, have been proposed to increase cardiovascular risk in a population-based study. ${ }^{33}$ Briefly, the metabolic profiling of the PEF group was in line with the pathological mechanism of endothelial dysfunction, but also depicted an aberrant pattern in genomic stability, protein synthesis, energy supply, and fatty acid mechanism. 
A

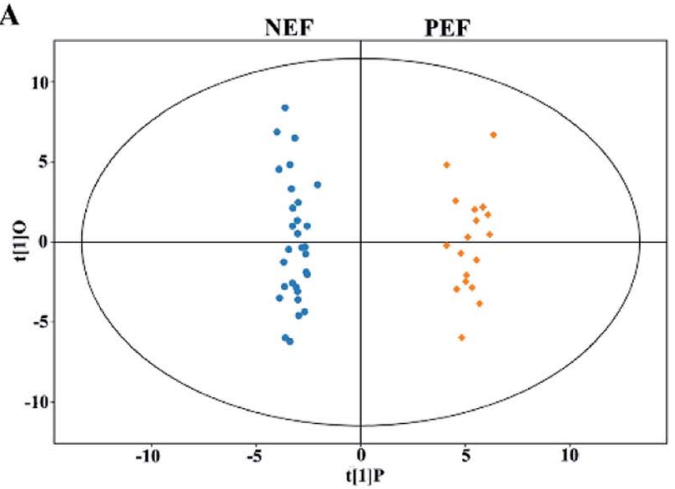

B

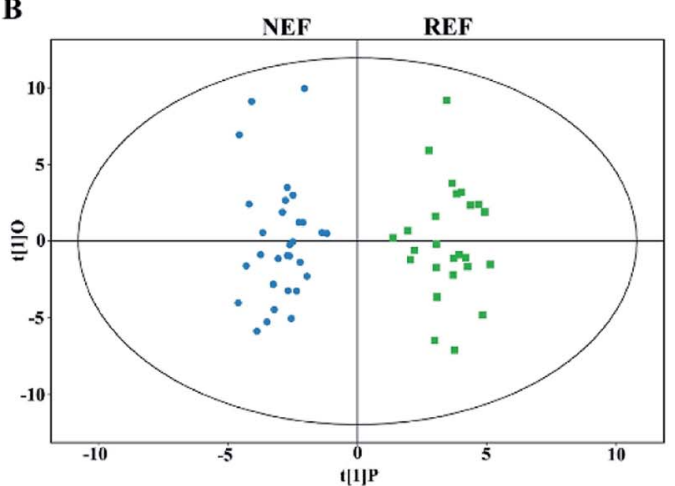

C

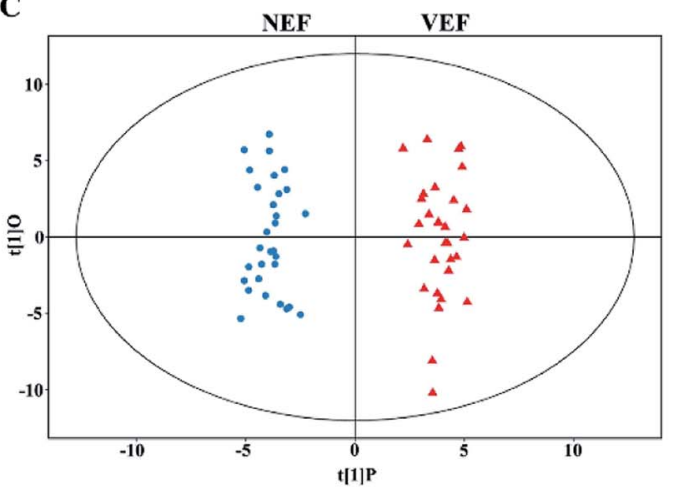

D

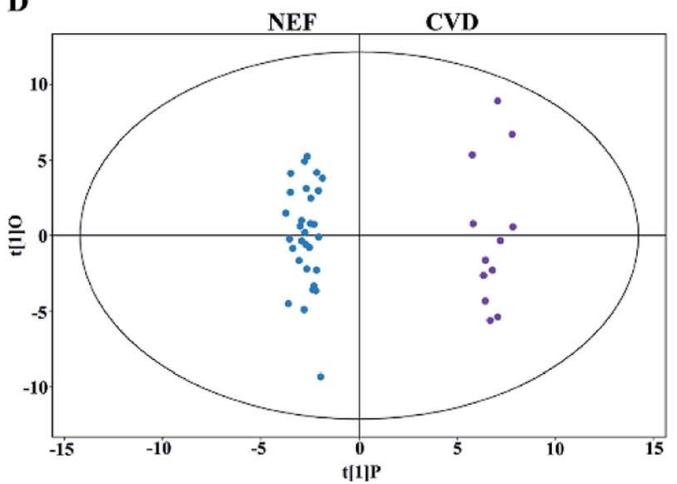

E

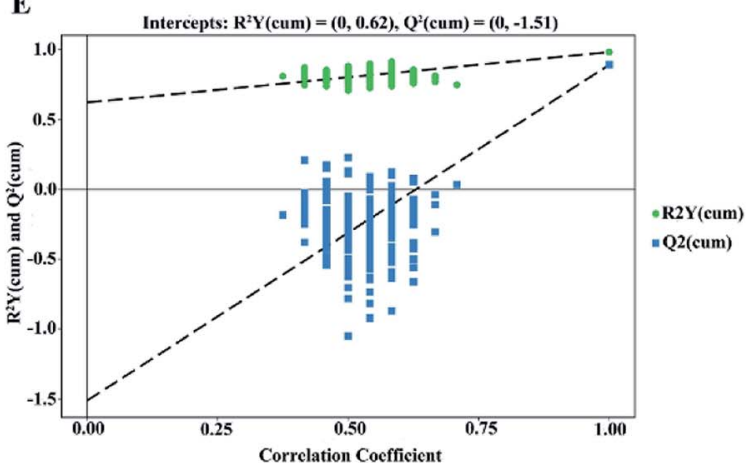

F

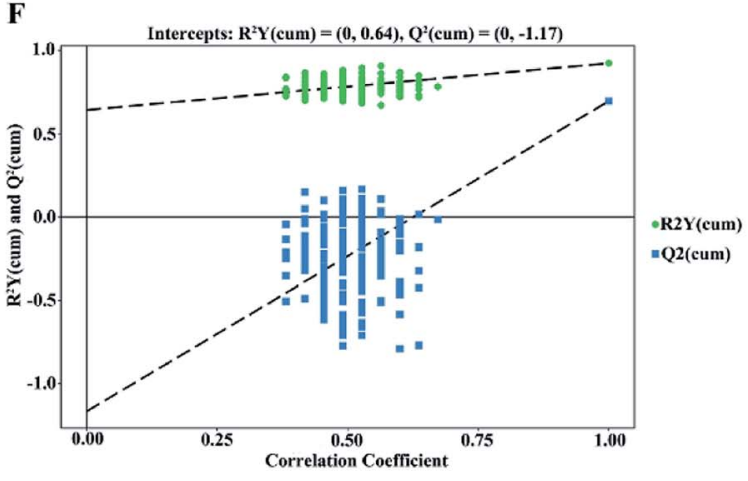

G

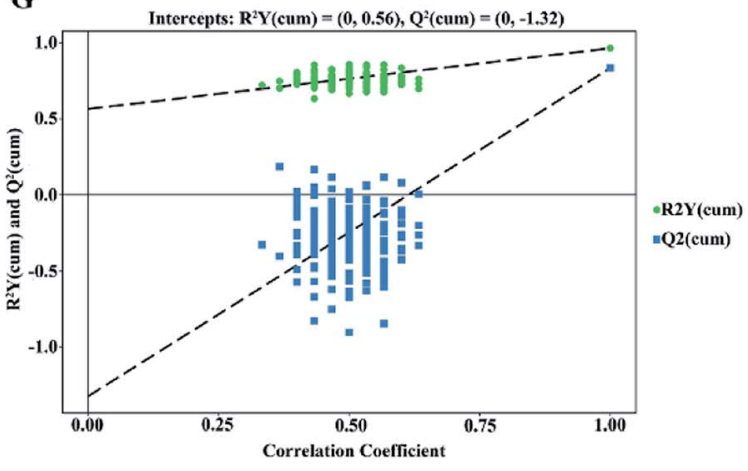

H

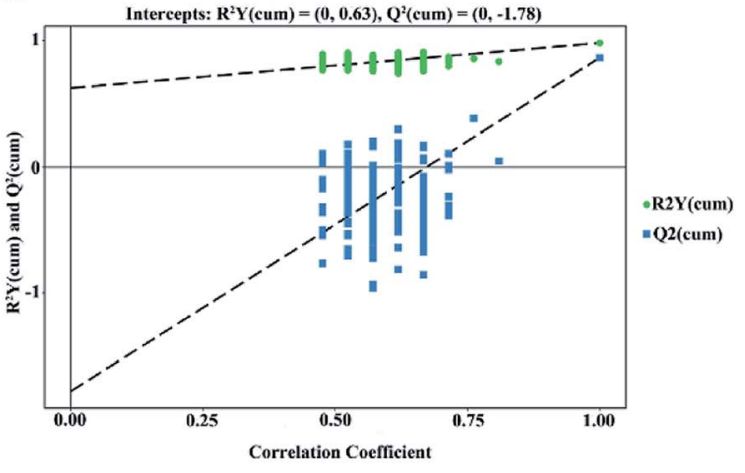

Fig. 3 The OPLS-DA score plots comparing (A) PEF versus NEF, (B) REF versus NEF, (C) VEF versus NEF and (D) CVD versus NEF. The corresponding 7-fold cross validations are shown in (E-H), respectively. OPLS-DA, orthogonal projection to latent structures-discriminant analysis; $\mathrm{NEF}$, normal endothelial function; PEF, cardiovascular risk-promoting endothelial function; REF, cardiovascular risk-resistant endothelial function; VEF, vulnerable endothelial function; CVD, cardiovascular disease. 
Table 1 Significantly different serum metabolites by comparison groups ${ }^{a}$

\begin{tabular}{|c|c|c|c|c|c|c|}
\hline Metabolites & Similarity & R.T. (minutes) & VIP & $P$ value & Fold change & Exclusive \\
\hline \multicolumn{7}{|l|}{ PEF versus $\mathrm{NEF}$} \\
\hline Tryptophan & 890 & 13.66 & 3.66 & $<0.001$ & $2.51 \times 10^{-9}$ & \\
\hline Monoolein & 778 & 15.93 & 1.03 & 0.023 & 2.21 & Yes \\
\hline 1-Monopalmitin & 775 & 15.12 & 1.02 & 0.033 & 2.11 & Yes \\
\hline Uric acid & 745 & 13.00 & 3.51 & $<0.001$ & $8.96 \times 10^{-9}$ & \\
\hline Oxamide & 475 & 8.77 & 1.20 & 0.045 & 1.28 & Yes \\
\hline Abietic acid & 467 & 14.37 & 3.42 & $<0.001$ & $1.50 \times 10^{-7}$ & \\
\hline 3-Hydroxynorvaline & 466 & 9.02 & 1.17 & 0.046 & 0.88 & Yes \\
\hline Thymidine & 455 & 10.65 & 2.41 & $<0.001$ & 0.08 & \\
\hline 2-Ketovaleric acid & 403 & 7.60 & 1.23 & 0.018 & 1.27 & Yes \\
\hline Phthalic acid & 393 & 11.02 & 1.03 & 0.010 & 1.80 & Yes \\
\hline \multicolumn{7}{|l|}{ REF versus NEF } \\
\hline Tagatose & 938 & 11.85 & 1.38 & $<0.001$ & 5.81 & Yes \\
\hline Arachidonic acid & 850 & 14.30 & 1.70 & 0.015 & 1.27 & Yes \\
\hline Arachidic acid & 681 & 14.55 & 2.62 & 0.007 & 0.41 & \\
\hline Sucrose & 656 & 15.30 & 4.08 & $<0.001$ & $6.30 \times 10^{-7}$ & \\
\hline Maleic acid & 649 & 8.59 & 1.87 & 0.011 & 1.23 & \\
\hline Oxalic acid & 541 & 7.28 & 1.53 & 0.016 & 0.91 & \\
\hline Phenyl beta-D-glucopyranoside & 480 & 14.01 & 1.75 & 0.030 & 1.32 & Yes \\
\hline Abietic acid & 467 & 14.37 & 3.89 & $<0.001$ & 0.09 & \\
\hline Itaconic acid & 446 & 8.88 & 1.26 & 0.021 & 0.80 & \\
\hline Pyrophosphate & 403 & 10.74 & 1.22 & 0.024 & 0.83 & \\
\hline \multicolumn{7}{|l|}{ VEF versus $\mathrm{NEF}$} \\
\hline Beta-mannosylglycerate & 965 & 11.80 & 1.23 & 0.032 & 1.15 & Yes \\
\hline Tryptophan & 890 & 13.66 & 2.90 & $<0.001$ & 0.33 & \\
\hline 2-Hydroxypyridine & 865 & 6.59 & 1.13 & 0.047 & 1.19 & Yes \\
\hline Urea & 793 & 8.19 & 2.38 & $<0.001$ & 3.80 & \\
\hline Uric acid & 745 & 13.00 & 3.59 & $<0.001$ & 0.07 & \\
\hline Asparagine & 713 & 10.84 & 1.31 & 0.010 & 1.23 & Yes \\
\hline Arachidic acid & 681 & 14.55 & 3.06 & $<0.001$ & 0.11 & \\
\hline Sucrose & 656 & 15.30 & 3.66 & $<0.001$ & $6.15 \times 10^{-7}$ & \\
\hline Maleic acid & 649 & 8.59 & 1.29 & 0.016 & 1.22 & \\
\hline Monostearin & 545 & 16.04 & 2.26 & 0.033 & 2.43 & Yes \\
\hline Oxalic acid & 541 & 7.28 & 1.25 & 0.016 & 0.90 & \\
\hline Uridine & 469 & 14.65 & 1.13 & $<0.001$ & 1.71 & Yes \\
\hline Abietic acid & 467 & 14.37 & 3.57 & $<0.001$ & $1.44 \times 10^{-7}$ & \\
\hline Itaconic acid & 446 & 8.88 & 1.52 & 0.003 & 0.78 & \\
\hline Pyrophosphate & 403 & 10.74 & 1.50 & 0.004 & 0.83 & \\
\hline Valine & 349 & 7.05 & 1.18 & 0.047 & 0.81 & Yes \\
\hline Lyxonic acid, 1,4-lactone & 346 & 11.27 & 2.31 & $<0.001$ & 1.56 & \\
\hline 5-Aminovaleric acid & 322 & 10.61 & 1.62 & 0.002 & 0.86 & \\
\hline Fructose & 299 & 11.95 & 2.48 & $<0.001$ & 0.14 & \\
\hline Thymol & 283 & 8.69 & 1.27 & 0.012 & 0.91 & Yes \\
\hline 4-Hydroxybutyrate & 242 & 8.12 & 1.34 & 0.030 & 1.08 & Yes \\
\hline 3-Ureidopropionate & 238 & 10.71 & 1.80 & $<0.001$ & 0.72 & \\
\hline
\end{tabular}

CVD versus $\mathrm{NEF}$ 
Table 1 (Contd.)

\begin{tabular}{|c|c|c|c|c|c|c|}
\hline Metabolites & Similarity & R.T. (minutes) & VIP & $P$ value & Fold change & Exclusive \\
\hline Tryptophan & 890 & 13.66 & 3.47 & $<0.001$ & $2.47 \times 10^{-9}$ & \\
\hline Urea & 793 & 8.19 & 3.02 & $<0.001$ & 6.81 & \\
\hline Uric acid & 745 & 13.00 & 3.30 & $<0.001$ & $8.82 \times 10^{-9}$ & \\
\hline Arachidic acid & 681 & 14.55 & 1.15 & 0.011 & 0.46 & \\
\hline Sucrose & 656 & 15.30 & 3.23 & $<0.001$ & $6.34 \times 10^{-7}$ & \\
\hline Lactic acid & 587 & 6.79 & 1.44 & 0.033 & 0.71 & Yes \\
\hline 3-Aminoisobutyric acid & 539 & 9.60 & 1.18 & 0.049 & 0.74 & \\
\hline 2-Monopalmitin & 487 & 14.95 & 2.43 & 0.005 & 0.15 & Yes \\
\hline Abietic acid & 467 & 14.37 & 3.13 & $<0.001$ & $1.48 \times 10^{-7}$ & \\
\hline Thymidine & 455 & 10.65 & 1.96 & $<0.001$ & 0.13 & \\
\hline Oxalacetic acid & 335 & 9.57 & 1.39 & 0.026 & 0.49 & Yes \\
\hline Glutamic acid & 315 & 10.59 & 1.27 & $<0.001$ & 0.23 & \\
\hline Fructose & 299 & 11.95 & 2.20 & $<0.001$ & 0.10 & \\
\hline
\end{tabular}

${ }^{a}$ Fold change with a value $>1$ indicates a higher level in each comparison group. The $P$ value was calculated using Student's $t$ test. VIP, variable importance in the projection.

The change of carbohydrate metabolites and derivatives was noticeable in the subjects with REF, especially in tagatose, a monosaccharide sweetener. In contrast to sucrose, tagatose has a very low glycemic index and has a protective effect on lipid profiles, glycemia, and atherosclerosis via inhibition of hepatic glycogenolysis, intestinal glucose absorption, or excess adiposity. ${ }^{34,35}$ In this study, the relative increase of low-calorie sugar in galactose metabolism may contribute to the protection of endothelial function even under high cardiovascular risk. In addition, arachidonic acid, a polyunsaturated fatty acid, was specifically elevated for the subjects with REF. Evidence (in vitro and in vivo) has shown that arachidonic acid can promote NO release via evoking $\mathrm{Ca}^{2+}$ signals ${ }^{36}$ and exert an antiinflammatory effect in the form of nitration, ${ }^{37}$ while impaired arachidonic acid metabolism can improve endothelial dysfunction. ${ }^{38}$ Therefore, the elevation of arachidonic acid may also be involved in the cardiovascular risk-resistant mechanism for the subjects with REF in this study.

For the subjects with VEF, the underlying mechanism remains poorly studied, although previous studies have suggested that unknown biological factors may contribute to atherosclerosis development even under a low-risk profile. ${ }^{1,39}$ Metabolite alterations of note, including the reduction of valine and 3-ureidopropionate and the elevation of maleic acid and 4-hydroxybutyrate, were involved in pantothenate and CoA biosynthesis (which are pivotal for CoA biosynthesis) and butanoate metabolism (which is pivotal for acetyl-CoA biosynthesis), ${ }^{40,41}$ thus supporting the relatively increased ratio of acetyl-CoA to CoA observed in the subjects with VEF. As the precursor of endogenous cholesterol biosynthesis, the abundance of acetyl-CoA is pertinent to not only cholesterol production but also eNOS function and oxidative stress through specific signaling pathways in vascular endothelial cells. ${ }^{22,43}$ Therefore, the dynamic imbalance between acetyl-CoA and CoA, characterized by relatively elevated acetyl-CoA, may contribute to endothelial dysfunction. In addition, although pyrimidine metabolism was disturbed in both the subjects with VEF and the subjects with PEF, in contrast with the depressed DNA metabolism observed in the subjects with PEF, perturbed RNA metabolism may exist in the VEF phenotype, as indicated by altered uridine and 3-ureidopropionate. Given the difference in genomic instability, further research is needed to determine the potential implications for the VEF phenotype.

CVD, the outcome of pathological development of vascular endothelial dysfunction, has been shown to have metabolic perturbations, such as decreased glycolysis and increased fatty acid oxidation in stable carotid plaques, ${ }^{12}$ a decrease in the tricarboxylic acid (TCA) cycle and phospholipid catabolism, higher branchedchain amino acid (BCAA) metabolites, and lower urea cycle metabolites in plasma samples. ${ }^{44,45}$ In this study, compared with the NEF group, lactic acid, oxalacetic acid and glutamic acid were substantially reduced in subjects with CVD. Lactic acid, a reduced product of pyruvate metabolism, is lower following decreased glycolytic flux; ${ }^{12}$ as a critical intermediate of the TCA cycle, oxalacetic acid can be derived from pyruvate via carboxylation, as well as from glutamic acid via glutamine oxidation, and plays a pivotal role in TCA cycle flux for tissue energy requirements. ${ }^{46,47}$ Therefore, these depressed metabolites may reflect the impaired energy metabolism and biosynthesis linked to the TCA cycle in CVD patients. Notably, we found that the metabolic profiles in the CVD patients, given the disturbed metabolic pathways, were mostly similar to the PEF group instead of the REF group or the VEF group, indicating the rationality of metabolic profiling in subjects with endothelial dysfunction.

Taken together, the metabolic profiles in the vascular endothelial dysfunction phenotype associated with high cardiovascular risk appear to mainly correlate with specific pathological changes. Meanwhile, disturbed galactose metabolism or an increased ratio of acetyl-CoA to CoA may contribute to the risk-resistant or vulnerable mechanism for vascular endothelial function. More importantly, for the primary or secondary care of cardiovascular disease, specific metabolite pools could be applied as potential circulating biomarkers to identify the individuals who may benefit from aggressive treatments despite low risk and those who should be provided less aggressive therapy despite high risk profiles.

Our study has several limitations. First, our subjects were not randomly sampled, and our findings should be replicated in 

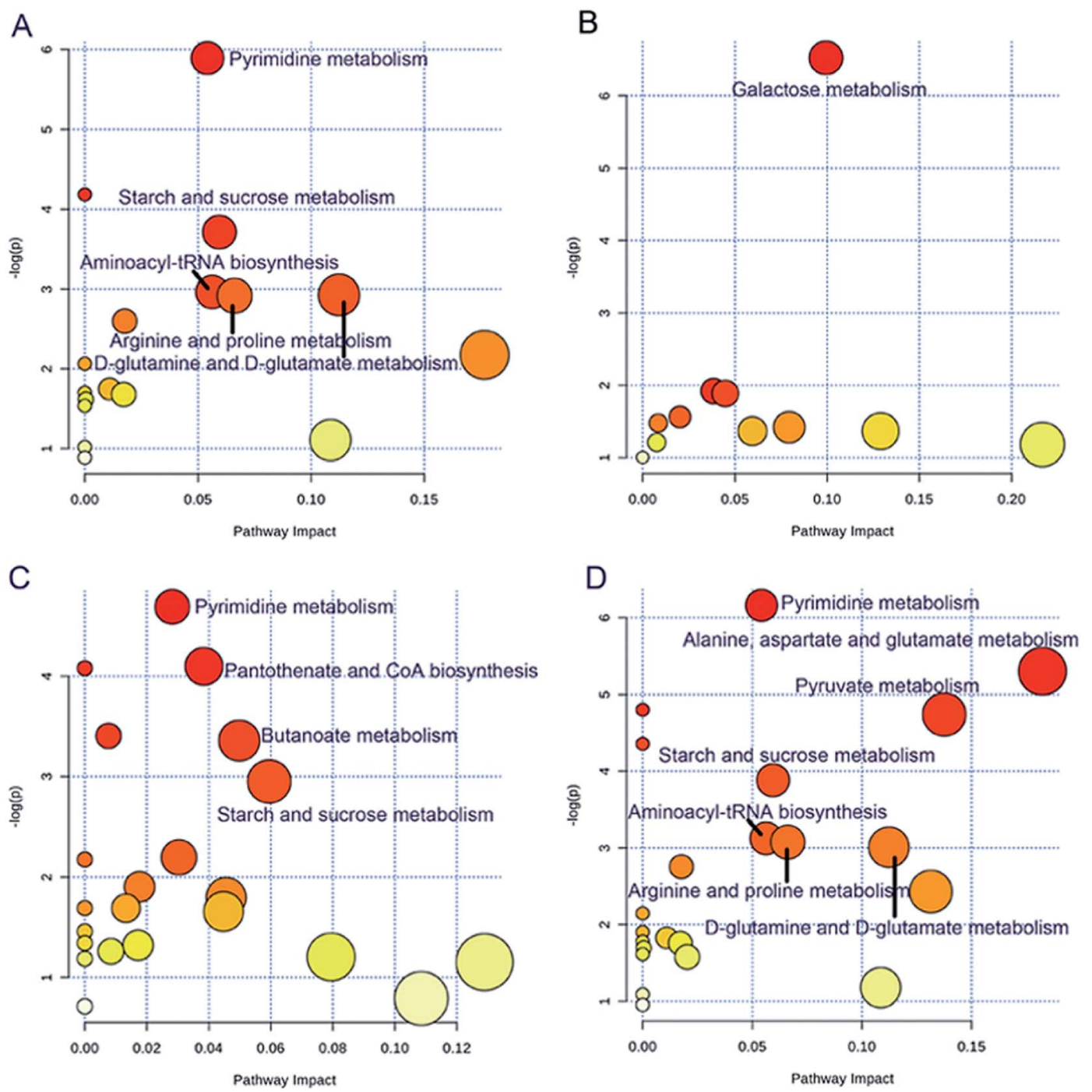

Fig. 4 The disturbed metabolic pathways showing various metabolic changes when comparing (A) PEF versus NEF, (B) REF versus NEF, (C) VEF versus NEF, and (D) CVD versus NEF. NEF, normal endothelial function; PEF, cardiovascular risk-promoting endothelial function; REF, cardiovascular risk-resistant endothelial function; VEF, vulnerable endothelial function; CVD, cardiovascular disease.

other larger populations. Second, although GC-TOF-MS has good separation efficiency and detection sensitivity, it was unable to detect all of the metabolites; thus, its detection of metabolites may be biased. Finally, bioinformation about some metabolites, such as abietic acid, phenyl beta-D-glucopyranoside, and $O$-methylthreonine, is still unavailable. Therefore, our findings should be carefully interpreted.

\section{Conclusion}

By characterizing the circulating metabolites in asymptomatic individuals and CVD patients, the current results clearly presented a diversity in metabolic patterns across different endothelial function phenotypes. From a metabolic standpoint, our findings may add to an understanding of the heterogeneous pathogenesis of early CVD in asymptomatic individuals with extreme cardiovascular risk.

\section{Author contributions}

Q. Z. conceived and designed the experiments; Q. Z., B. M. and Y. Y. wrote the manuscript; B. M., Y. Y., Q. M. and C. Y. performed the experiments; Q. Z., B. M. and Y. Y. analyzed the data. All authors have read and approved the final manuscript.

\section{Conflicts of interest}

There are no conflicts of interest to declare.

\section{Acknowledgements}

This study was supported by the Natural Science Foundation of China (grants 81360422 and 81860570) and the Guangxi Natural Science Foundation (grants 2014GXNSFAA118167 and 2016GXNSFAA380050). 


\section{References}

1 M. Magnoni, D. Andreini, M. Gorini, T. Moccetti, M. G. Modena, M. Canestrari, S. Berti, G. Casolo, D. Gabrielli, P. Marraccini, G. Pontone, S. Masson, R. Latini, A. P. Maggioni, A. Maseri and C. S. Group, Coronary atherosclerosis in outlier subjects at the opposite extremes of traditional risk factors: rationale and preliminary results of the coronary atherosclerosis in outlier subjects: protective and novel individual risk factors evaluation (CAPIRE) study, Am. Heart J., 2016, 173, 18-26.

2 M. G. Silverman, M. J. Blaha, H. M. Krumholz, M. J. Budoff, R. Blankstein, C. T. Sibley, A. Agatston, R. S. Blumenthal and K. Nasir, Impact of coronary artery calcium on coronary heart disease events in individuals at the extremes of traditional risk factor burden: the Multi-Ethnic Study of Atherosclerosis, Eur. Heart J., 2014, 35(33), 2232-2241.

3 U. N. Khot, M. B. Khot, C. T. Bajzer, S. K. Sapp, E. M. Ohman, S. J. Brener, S. G. Ellis, A. M. Lincoff and E. J. Topol, Prevalence of conventional risk factors in patients with coronary heart disease, JAMA, 2003, 290(7), 898-904.

4 F. F. Faletra, C. Klersy, I. D'Angeli, M. Penco, V. Procaccini, E. Pasotti, A. Marcolongo, G. B. Pedrazzini, S. De Castro, M. Scappaticci, T. Moccetti and A. Auricchio, Relation between coronary atherosclerotic plaques and traditional risk factors in people with no history of cardiovascular disease undergoing multi-detector computed coronary angiography, Heart, 2009, 95(15), 1265-1272.

5 E. Maffei, C. Martini, S. Seitun, T. Arcadi, C. Tedeschi, A. Guaricci, R. Malago, G. Tarantini, A. Aldrovandi and F. Cademartiri, Computed tomography coronary angiography in the selection of outlier patients: a feasibility report, Radiol. Med., 2012, 117(2), 214-229.

6 K. J. Kim, S. I. Choi, M. S. Lee, J. A. Kim, E. J. Chun and C. H. Jeon, The prevalence and characteristics of coronary atherosclerosis in asymptomatic subjects classified as low risk based on traditional risk stratification algorithm: assessment with coronary CT angiography, Heart, 2013, 99(15), 1113-1117.

7 R. M. Stoekenbroek, S. M. Boekholdt, R. Luben, G. K. Hovingh, A. H. Zwinderman, N. J. Wareham, K. T. Khaw and R. J. Peters, Heterogeneous impact of classic atherosclerotic risk factors on different arterial territories: the EPIC-Norfolk prospective population study, Eur. Heart J., 2016, 37(11), 880-889.

8 M. A. Gimbrone Jr and G. Garcia-Cardena, Endothelial Cell Dysfunction and the Pathobiology of Atherosclerosis, Circ. Res., 2016, 118(4), 620-636.

9 E. Gutierrez, A. J. Flammer, L. O. Lerman, J. Elizaga, A. Lerman and F. Fernandez-Aviles, Endothelial dysfunction over the course of coronary artery disease, Eur. Heart J., 2013, 34(41), 3175-3181.

10 A. J. Flammer, T. Anderson, D. S. Celermajer, M. A. Creager, J. Deanfield, P. Ganz, N. M. Hamburg, T. F. Luscher, M. Shechter, S. Taddei, J. A. Vita and A. Lerman, The assessment of endothelial function: from research into clinical practice, Circulation, 2012, 126(6), 753-767.

11 G. Eelen, P. de Zeeuw, M. Simons and P. Carmeliet, Endothelial cell metabolism in normal and diseased vasculature, Circ. Res., 2015, 116(7), 1231-1244.

12 L. Tomas, A. Edsfeldt, I. G. Mollet, L. Perisic Matic, C. Prehn, J. Adamski, G. Paulsson-Berne, U. Hedin, J. Nilsson, E. Bengtsson, I. Goncalves and H. Bjorkbacka, Altered metabolism distinguishes high-risk from stable carotid atherosclerotic plaques, Eur. Heart J., 2018, 39(24), 23012310.

13 D. Vojinovic, S. J. van der Lee, C. M. van Duijn, M. W. Vernooij, M. Kavousi, N. Amin, A. Demirkan, M. A. Ikram, A. van der Lugt and D. Bos, Metabolic profiling of intra- and extracranial carotid artery atherosclerosis, Atherosclerosis, 2018, 272, 60-65.

14 N. P. Paynter, R. Balasubramanian, F. Giulianini, D. D. Wang, L. F. Tinker, S. Gopal, A. A. Deik, K. Bullock, K. A. Pierce, J. Scott, M. A. Martinez-Gonzalez, R. Estruch, J. E. Manson, N. R. Cook, C. M. Albert, C. B. Clish and K. M. Rexrode, Metabolic Predictors of Incident Coronary Heart Disease in Women, Circulation, 2018, 137(8), 841-853.

15 A. Floegel, T. Kuhn, D. Sookthai, T. Johnson, C. Prehn, U. Rolle-Kampczyk, W. Otto, C. Weikert, T. Illig, M. von Bergen, J. Adamski, H. Boeing, R. Kaaks and T. Pischon, Serum metabolites and risk of myocardial infarction and ischemic stroke: a targeted metabolomic approach in two German prospective cohorts, Eur. J. Epidemiol., 2018, 33(1), 55-66.

16 T. F. Tian, S. Y. Wang, T. C. Kuo, C. E. Tan, G. Y. Chen, C. H. Kuo, C. S. Chen, C. C. Chan, O. A. Lin and Y. J. Tseng, Web Server for Peak Detection, Baseline Correction, and Alignment in Two-Dimensional Gas Chromatography Mass Spectrometry-Based Metabolomics Data, Anal. Chem., 2016, 88(21), 10395-10403.

17 C. Sales, M. I. Cervera, R. Gil, T. Portoles, E. Pitarch and J. Beltran, Quality classification of Spanish olive oils by untargeted gas chromatography coupled to hybrid quadrupole-time of flight mass spectrometry with atmospheric pressure chemical ionization and metabolomics-based statistical approach, Food Chem., 2017, 216, 365-373.

18 R. B. D'Agostino Sr, R. S. Vasan, M. J. Pencina, P. A. Wolf, M. Cobain, J. M. Massaro and W. B. Kannel, General cardiovascular risk profile for use in primary care: the Framingham Heart Study, Circulation, 2008, 117(6), 743-753.

19 T. Maruhashi, J. Soga, N. Fujimura, N. Idei, S. Mikami, Y. Iwamoto, M. Kajikawa, T. Matsumoto, T. Hidaka, Y. Kihara, K. Chayama, K. Noma, A. Nakashima, C. Goto, H. Tomiyama, B. Takase, A. Yamashina and Y. Higashi, Relationship between flow-mediated vasodilation and cardiovascular risk factors in a large community-based study, Heart, 2013, 99(24), 1837-1842.

20 Q. Zhong, Q. Nong, B. Mao, X. Pan and L. Meng, Association of Impaired Vascular Endothelial Function with Increased Cardiovascular Risk in Asymptomatic Adults, BioMed Res. Int., 2018, 2018, 3104945. 
21 T. Maruhashi, Y. Iwamoto, M. Kajikawa, N. Oda, S. Kishimoto, S. Matsui, H. Hashimoto, Y. Aibara, F. M. Yusoff, T. Hidaka, Y. Kihara, K. Chayama, K. Noma, A. Nakashima, C. Goto, E. Hida and Y. Higashi, Interrelationships Among Flow-Mediated Vasodilation, Nitroglycerine-Induced Vasodilation, Baseline Brachial Artery Diameter, Hyperemic Shear Stress, and Cardiovascular Risk Factors, J. Am. Heart Assoc., 2017, 7(1), e006797.

22 M. A. Kluge, J. L. Fetterman and J. A. Vita, Mitochondria and endothelial function, Circ. Res., 2013, 112(8), 1171-1188.

23 N. E. Deutz, The 2007 ESPEN Sir David Cuthbertson Lecture: amino acids between and within organs. The glutamateglutamine-citrulline-arginine pathway, Clin. Nutr., 2008, 27(3), 321-327.

24 A. Popolo, S. Adesso, A. Pinto, G. Autore and S. Marzocco, LArginine and its metabolites in kidney and cardiovascular disease, Amino Acids, 2014, 46(10), 2271-2286.

25 M. D'Apolito, X. Du, D. Pisanelli, M. Pettoello-Mantovani, A. Campanozzi, F. Giacco, A. B. Maffione, A. L. Colia, M. Brownlee and I. Giardino, Urea-induced ROS cause endothelial dysfunction in chronic renal failure, Atherosclerosis, 2015, 239(2), 393-400.

26 Y. Itahana, R. Han, S. Barbier, Z. Lei, S. Rozen and K. Itahana, The uric acid transporter SLC2A9 is a direct target gene of the tumor suppressor p53 contributing to antioxidant defense, Oncogene, 2015, 34(14), 1799-1810.

27 C. X. Shi, M. X. Zhao, X. D. Shu, X. Q. Xiong, J. J. Wang, X. Y. Gao, Q. Chen, Y. H. Li, Y. M. Kang and G. Q. Zhu, Beta-aminoisobutyric acid attenuates hepatic endoplasmic reticulum stress and glucose/lipid metabolic disturbance in mice with type 2 diabetes, Sci. Rep., 2016, 6, 21924.

28 G. Liu, S. Chen, J. Zhong, K. Teng and Y. Yin, Crosstalk between Tryptophan Metabolism and Cardiovascular Disease, Mechanisms, and Therapeutic Implications, Oxid. Med. Cell. Longevity, 2017, 2017, 1602074.

29 C. Murr, T. B. Grammer, M. E. Kleber, A. Meinitzer, W. Marz and D. Fuchs, Low serum tryptophan predicts higher mortality in cardiovascular disease, Eur. J. Clin. Invest., 2015, 45(3), 247-254.

30 D. Qiu, L. Huang and S. Lin, Cryptophyte farming by symbiotic ciliate host detected in situ, Proc. Natl. Acad. Sci. U. S. A., 2016, 113(43), 12208-12213.

31 P. A. Vorkas, J. Shalhoub, G. Isaac, E. J. Want, J. K. Nicholson, E. Holmes and A. H. Davies, Metabolic phenotyping of atherosclerotic plaques reveals latent associations between free cholesterol and ceramide metabolism in atherogenesis, J. Proteome Res., 2015, 14(3), 1389-1399.

32 Y. L. Pang, K. Poruri and S. A. Martinis, tRNA synthetase: tRNA aminoacylation and beyond, Wiley Interdiscip. Rev.: RNA, 2014, 5(4), 461-480.

33 C. Stegemann, R. Pechlaner, P. Willeit, S. R. Langley, M. Mangino, U. Mayr, C. Menni, A. Moayyeri, P. Santer, G. Rungger, T. D. Spector, J. Willeit, S. Kiechl and M. Mayr, Lipidomics profiling and risk of cardiovascular disease in the prospective population-based Bruneck study, Circulation, 2014, 129(18), 1821-1831.

34 S. B. Police, J. C. Harris, R. A. Lodder and L. A. Cassis, Effect of diets containing sucrose vs. D-tagatose in hypercholesterolemic mice, Obesity, 2009, 17(2), 269-275.

35 M. Guerrero-Wyss, S. Duran Aguero and L. Angarita Davila, D-Tagatose is a Promising Sweetener to Control Glycaemia: A New Functional Food, BioMed Res. Int., 2018, 2018, 8718053.

36 E. Zuccolo, S. Dragoni, V. Poletto, P. Catarsi, D. Guido, A. Rappa, M. Reforgiato, F. Lodola, D. Lim, V. Rosti, G. Guerra and F. Moccia, Arachidonic acid-evoked $\mathrm{Ca}(2+)$ signals promote nitric oxide release and proliferation in human endothelial colony forming cells, Vasc. Pharmacol., 2016, 87, 159-171.

37 A. Trostchansky and H. Rubbo, Anti-inflammatory signaling actions of electrophilic nitro-arachidonic acid in vascular cells and astrocytes, Arch. Biochem. Biophys., 2017, 617, 155-161.

38 A. Sanchez, C. Contreras, N. Villalba, P. Martinez, A. C. Martinez, A. Briones, M. Salaices, A. Garcia-Sacristan, M. Hernandez and D. Prieto, Altered arachidonic acid metabolism via COX-1 and COX-2 contributes to the endothelial dysfunction of penile arteries from obese Zucker rats, Br. J. Pharmacol., 2010, 159(3), 604-616.

39 I. S. Santos, A. P. Alencar, T. Rundek, A. C. Goulart, S. M. Barreto, A. C. Pereira, I. M. Bensenor and P. A. Lotufo, Low Impact of Traditional Risk Factors on Carotid Intima-Media Thickness: The ELSA-Brasil Cohort, Arterioscler., Thromb., Vasc. Biol., 2015, 35(9), 2054-2059.

40 I. Di Meo, M. Carecchio and V. Tiranti, Inborn errors of coenzyme A metabolism and neurodegeneration, $J$. Inherited Metab. Dis., 2019, 42(1), 49-56.

41 S. Tagore and R. K. De, Evolutionary growth of certain metabolic pathways involved in the functioning of GAD and INS genes in type 1 diabetes mellitus: their architecture and stability, Comput. Biol. Med., 2015, 61, 1935.

42 A. Pircher, L. Treps, N. Bodrug and P. Carmeliet, Endothelial cell metabolism: a novel player in atherosclerosis? Basic principles and therapeutic opportunities, Atherosclerosis, 2016, 253, 247-257.

43 A. Oesterle, U. Laufs and J. K. Liao, Pleiotropic Effects of Statins on the Cardiovascular System, Circ. Res., 2017, 120(1), 229-243.

44 Y. Fan, Y. Li, Y. Chen, Y. J. Zhao, L. W. Liu, J. Li, S. L. Wang, R. N. Alolga, Y. Yin, X. M. Wang, D. S. Zhao, J. H. Shen, F. Q. Meng, X. Zhou, H. Xu, G. P. He, M. D. Lai, P. Li, W. Zhu and L. W. Qi, Comprehensive Metabolomic Characterization of Coronary Artery Diseases, J. Am. Coll. Cardiol., 2016, 68(12), 1281-1293.

45 S. H. Shah, W. E. Kraus and C. B. Newgard, Metabolomic profiling for the identification of novel biomarkers and mechanisms related to common cardiovascular diseases: form and function, Circulation, 2012, 126(9), 1110-1120.

46 S. Christen, D. Lorendeau, R. Schmieder, D. Broekaert, K. Metzger, K. Veys, I. Elia, J. M. Buescher, M. F. Orth, 
S. M. Davidson, T. G. Grunewald, K. De Bock and S. M. Fendt, Breast Cancer-Derived Lung Metastases Show Increased Pyruvate Carboxylase-Dependent Anaplerosis, Cell. Reprogram., 2016, 17(3), 837-848.

47 C. Yang, B. Ko, C. T. Hensley, L. Jiang, A. T. Wasti, J. Kim, J. Sudderth, M. A. Calvaruso, L. Lumata, M. Mitsche,
J. Rutter, M. E. Merritt and R. J. DeBerardinis, Glutamine oxidation maintains the TCA cycle and cell survival during impaired mitochondrial pyruvate transport, Mol. Cell, 2014, 56(3), 414-424. 